\title{
Ventricular Wall of the Heart
}

National Cancer Institute

\section{Source}

National Cancer Institute. Ventricular Wall of the Heart. NCI Thesaurus. Code C119295.

The tissue layers that form the cardiac ventricle. They include the endocardium,

myocardium and pericardium. 